\title{
Optimize your treatment of endometriosis by using an FDA-approved hormonal medication
}

\author{
For women with endometriosis, if one estrogen-progestin contraceptive \\ results in suboptimal control of pelvic pain, do not prescribe a different \\ brand of the same contraceptive type. In this situation, prescribe one of the \\ US Food and Drug Administration (FDA) approved treatments for pelvic pain \\ caused by endometriosis.
}

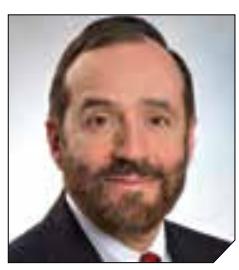

\author{
Robert L. Barbieri, MD \\ Chair Emeritus, Department of Obstetrics and Gynecology \\ Interim Chief, Obstetrics \\ Brigham and Women's Hospital \\ Kate Macy Ladd Distinguished Professor of Obstetrics, \\ Gynecology and Reproductive Biology \\ Harvard Medical School \\ Boston, Massachusetts
}

W omen with endometriosis often present for medical care for one or more of the following health issues: pelvic pain, infertility, and/or an adnexal cyst (endometrioma). For women with moderate or severe pelvic pain and laparoscopically diagnosed endometriosis, hormone therapy is often necessary to achieve maximal longterm reduction in pain and optimize health. I focus on opportunities to optimize hormonal treatment of endometriosis in this editorial.

\section{When plan $A$ is not working, move expeditiously to plan B}

Cyclic or continuous combination estrogen-progestin contraceptives are commonly prescribed to treat pelvic pain caused by endometriosis. Although endometriosis pain may

doi: $10.12788 /$ obgm.0084 initially improve with estrogen-progestin contraceptives, many women on this medication will eventually report that they have worsening pelvic pain that adversely impacts their daily activities. Surprisingly, clinicians often continue to prescribe estrogen-progestin contraceptives even after the patient reports that the treatment is not effective, and their pain continues to be bothersome.

Patients benefit when they have access to the full range of hormone treatments that have been approved by the FDA for the treatment of moderate to severe pelvic pain caused by endometriosis (TABLE). In the situation where an estrogen-progestin contraceptive is no longer effective at reducing the pelvic pain, I will often offer the patient the option of norethindrone acetate (NEA) or elagolix treatment. My experience is that stopping the estrogen-progestin contraceptive and starting NEA or elagolix will result in a significant decrease in pain symptoms and improvement in the patient's quality of life.

Other FDA-approved options to treat pelvic pain caused by endometriosis include depot medroxyprogesterone acetate injectable suspension, depot leuprolide acetate, goserelin implant, and danazol. I do not routinely prescribe depot medroxyprogesterone acetate because some patients report new onset or worsening symptoms of depression on the medication. I prescribe depot-leuprolide acetate less often than in the past, because many patients report moderate to severe hypoestrogenic symptoms on this medication. In women taking depotleuprolide acetate, moderate to severe vasomotor symptoms can be improved by prescribing NEA pills, but the alternative of norethindrone monotherapy is less expensive. I seldom use goserelin or danazol in my practice. The needle required 
TABLE Hormone treatments approved by the FDA to treat moderate to severe pain caused by endometriosis

\begin{tabular}{|c|c|c|c|}
\hline FDA-approved medication & Route of administration & Dose & Relative cost ${ }^{a}$ \\
\hline \multicolumn{4}{|l|}{ Progestins } \\
\hline Norethindrone acetate & Oral & 5 mg daily & $\$$ \\
\hline $\begin{array}{l}\text { Medroxyprogesterone acetate } \\
\text { injectable suspension }\end{array}$ & Subcutaneous injection & 104 mg every 3 months & $\$ \$$ \\
\hline \multicolumn{4}{|l|}{ GnRH analogues } \\
\hline Nafarelin acetate & Intranasal spray & One spray twice daily & $\$ \$ \$ \$$ \\
\hline Depot-leuprolide acetate & Intramuscular injection & 3.75 mg monthly & $\$ \$ \$ \$$ \\
\hline Goserelin & Subcutaneous implant & 3.6 mg monthly & $\$ \$ \$ \$$ \\
\hline Elagolix & Oral & $150 \mathrm{mg}$ daily or $200 \mathrm{mg}$ twice daily & $\$ \$ \$ \$$ \\
\hline \multicolumn{4}{|l|}{ Androgens } \\
\hline Danazol & Oral & 200 mg twice daily & $\$ \$ \$$ \\
\hline
\end{tabular}

to place the goserelin implant has a diameter of approximately $1.7 \mathrm{~mm}$ (16 gauge) or $2.1 \mathrm{~mm}$ (14 gauge), for the $3.6 \mathrm{mg}$ and $10 \mathrm{mg}$ doses, respectively. The large diameter of the needle can cause pain and bruising at the implant site. As a comparison, the progestin subdermal implant needle is approximately $2.1 \mathrm{~mm}$ in diameter. Danazol is associated with weight gain, and most women prefer to avoid this side effect.

\section{Norethindrone acetate}

NEA $5 \mathrm{mg}$ daily is approved by the FDA to treat endometriosis. ${ }^{1}$ NEA was approved at a time when large controlled clinical trials were not routinely required for a medicine to be approved. The data to support NEA treatment of pelvic pain caused by endometriosis is based on cohort studies. In a study of 194 women, median age 21 years with moderate to severe pelvic pain and surgically proven endometriosis, the effect of NEA on pelvic pain was explored. $^{2}$ The initial dose of NEA was $5 \mathrm{mg}$ daily. If the patient did not achieve a reduction in pelvic pain and amenorrhea on the NEA dose of $5 \mathrm{mg}$ daily, the dose was increased by $2.5 \mathrm{mg}$ every 2 weeks, up to a maximum of $15 \mathrm{mg}$, until amenorrhea and/or a decrease in pelvic pain was achieved. Ninety-five percent of the women in this cohort had previously been treated with an estrogen-progestin contraceptive or a GnRH antagonist and had discontinued those medications because of inadequate control of pelvic pain or because of side effects of the medication.

In this large cohort, $65 \%$ of women reported significant improvement in pelvic pain, with a median pain score of 5 before treatment and 0 following NEA treatment. About $55 \%$ of the women reported no side effects. The most commonly reported side effects were weight gain (16\%; mean weight gain, $3.1 \mathrm{~kg}$ ), acne (10\%), mood lability (9\%), hot flashes (8\%), depression (6\%), scalp hair loss (4\%), headache (4\%), nausea (3\%), and deepening of the voice (1\%). (In this study women could report more than one side effect.)

In another cohort study of 52 women with pelvic pain and surgically confirmed endometriosis, NEA treatment resulted in pain relief in $94 \%$ of the women. ${ }^{3}$ Breakthrough bleeding was a common side effect, reported by $58 \%$ of participants. The investigators concluded that NEA treatment was a "cost-effective alternative with relatively mild side effects in the treatment of symptomatic endometriosis." A conclusion which I endorse.

NEA has been reported to effectively treat ovarian endometriomas and rectovaginal endometriosis., ${ }^{4,5}$ In a cohort of 18 women who had previously had the surgical resection of an ovarian endometriosis cyst and had postoperative recurrence of pelvic pain and ovarian endometriosis, treatment was initiated with an 
escalating NEA regimen. ${ }^{4}$ Treatment was initiated with NEA $5 \mathrm{mg}$ daily, with the dosage increased every 2 weeks by $2.5 \mathrm{mg}$ until amenorrhea was established. Most women achieved amenorrhea with NEA $5 \mathrm{mg}$ daily, and $89 \%$ had reduced pelvic pain. The investigators reported complete regression of the endometriosis cyst(s) in $74 \%$ of the women. In my experience, NEA does not result in complete regression of endometriosis cysts, but it does cause a reduction in cyst diameter and total volume.

In a retrospective cohort study, 61 women with pelvic pain and rectovaginal endometriosis had 5 years of treatment with NEA $2.5 \mathrm{mg}$ or $5.0 \mathrm{mg}$ daily. ${ }^{5} \mathrm{NEA}$ treatment resulted in a decrease in dysmenorrhea, deep dyspareunia, and dyschezia. The most common side effects attributed to NEA treatment were weight gain $(30 \%)$, vaginal bleeding (23\%), decreased libido (11\%), headache (9\%), bloating or swelling (8\%), depression (7\%), and acne (5\%). In women who had sequential imaging studies, NEA treatment resulted in a decrease in rectovaginal lesion volume, stable disease volume, or an increase in lesion volume in $56 \%, 32 \%$, and $12 \%$ of the women, respectively. The investigators concluded that for women with rectovaginal endometriosis, NEA treatment is a low-cost option for long-term treatment.

In my practice, I do not prescribe NEA at doses greater than 5 mg daily. There are case reports that NEA at a dose of $\geq 10 \mathrm{mg}$ daily is associated with the development of a hepatic adenoma, ${ }^{6}$ elevated liver transaminase concentration, ${ }^{7}$ and jaundice. ${ }^{8}$ If NEA $5 \mathrm{mg}$ daily is not effective in controlling pelvic pain caused by endometriosis, I stop the NEA and start a GnRH analogue, most often elagolix.

NEA $5 \mathrm{mg}$ is not FDA approved as a contraceptive. However, norethindrone $0.35 \mathrm{mg}$ daily, also known as the "mini-pill", is approved as a progestin-only contraceptive. ${ }^{9}$ NEA is rapidly and completely deacetylated to norethindrone, and the disposition of oral NEA is indistinguishable from that of norethindrone. ${ }^{1}$ Since norethindrone $0.35 \mathrm{mg}$ daily is approved as a contraceptive, it is highly likely that NEA $5 \mathrm{mg}$ has contraceptive properties if taken daily.

\section{Elagolix}

Elagolix is FDA approved for the treatment of pelvic pain caused by endometriosis. I reviewed the key studies resulting in FDA approval in the November 2018 issue of OBG MANAGEMENT. ${ }^{10}$

In the Elaris Endometriosis-I study, 872 women with endometriosis and pelvic pain were randomly assigned to treatment with 1 of 2 doses of elagolix (high-dose [200 mg twice daily] and low-dose [150 mg once daily]) or placebo. ${ }^{11}$ After 3 months of therapy, a clinically meaningful reduction in dysmenorrhea pain was reported by $76 \%, 46 \%$, and $20 \%$ of the women in the highdose elagolix, low-dose elagolix, and placebo groups, respectively $(P<.001$ for comparisons of elagolix to placebo). After 3 months of therapy, a clinically meaningful reduction in nonmenstrual pain or decreased or stable use of rescue analgesics was reported by $55 \%, 50 \%$, and $37 \%$ of the women in the high-dose elagolix, low-dose elagolix, and placebo groups, respectively $(P<.01$ low-dose elagolix vs placebo and $P<.001$ highdose elagolix vs placebo).

Hot flashes that were severe enough to be reported as an adverse event by the study participants were reported by $42 \%, 24 \%$, and $7 \%$ of the women in the high-dose elagolix, low-dose elagolix, and placebo groups. Bone density was measured at baseline and after 6 months of treatment. Lumbar bone density changes were $-2.61 \%,-0.32 \%$, and $+0.47 \%$ and hip femoral neck bone density changes were $-1.89 \%,-0.39 \%$, and $+0.02 \%$ in the high-dose elagolix, low-dose elagolix, and placebo groups, respectively.

Another large clinical trial of elagolix for the treatment of pelvic pain caused by endometriosis, Elaris EM-II, involving 817 women, produced results very similar to those reported in Elaris EM-I. The elagolix continuation studies, Elaris EM-III and -IV, demonstrated efficacy and safety of elagolix through 12 months of treatment. ${ }^{12}$

In my 2018 review, ${ }^{10}$ I noted that elagolix dose adjustment can be utilized to attempt to achieve maximal pain relief with minimal vasomotor symptoms. Elagolix at $200 \mathrm{mg}$ twice daily produces a mean estradiol concentration of $12 \mathrm{pg} / \mathrm{mL}$, whereas elagolix at $150 \mathrm{mg}$ daily resulted in a mean estradiol concentration of $41 \mathrm{pg} / \mathrm{mL} \cdot{ }^{13}$ The estrogen threshold hypothesis posits that in women with endometriosis a stable estradiol concentration of 20 to $30 \mathrm{pg} / \mathrm{mL}$ is often associated with decreased pain and fewer vasomotor events. ${ }^{14}$ To achieve the target estradiol range of 20 to $30 \mathrm{pg} / \mathrm{mL}$, I often initiate elagolix treatment with $200 \mathrm{mg}$ twice daily. This enables a rapid onset of amenorrhea and a reduction in pelvic pain. Once amenorrhea has been achieved and a decrease in pelvic pain has occurred, I adjust the dose downward to $200 \mathrm{mg}$ twice daily on even calendar days of each month and $200 \mathrm{mg}$ once daily on odd calendar days each month. Some women will have continued pain relief and amenorrhea when the dose is further decreased to $200 \mathrm{mg}$ once daily. If bothersome bleeding recurs and/ or pain symptoms increase in 
severity, the dose can be increased to $200 \mathrm{mg}$ twice daily or an alternating regimen of $200 \mathrm{mg}$ twice daily and $200 \mathrm{mg}$ once daily, every 2 days. An alternative to dose adjustment is to combine elagolix with NEA, which can reduce the severity of hot flashes and reduce bone loss caused by hypoestrogenism. ${ }^{15,16}$

Health insurers and pharmacy benefits managers may require a prior authorization before approving and dispensing elagolix. The prior authorization process can be burdensome for clinicians, consuming limited healthcare resources, contributing to burnout and frustrating patients. ${ }^{17}$ Elagolix is less expensive than depotleuprolide acetate and nafarelin nasal spray and somewhat more expensive than a goserelin implant. ${ }^{18,19}$

Elagolix is not approved as a contraceptive. In the Elaris EM-I and -II trials women were advised to use 2 forms of contraception, although pregnancies did occur. There were 6 pregnancies among 475 women taking elagolix $150 \mathrm{mg}$ daily and 2 pregnancies among 477 women taking elagolix $200 \mathrm{mg}$ twice daily. ${ }^{20}$ Women taking elagolix should be advised to use a contraceptive, but not an estrogen-progestin contraceptive.

\section{Do not use opioids to treat chronic pelvic pain caused by endometriosis}

One of the greatest public health tragedies of our era is the opioid misuse epidemic. Hundreds of thousands of deaths have been caused by opioid misuse. The Centers for Disease Control and Prevention reported that for the 12-month period ending in May 2020, there were 81,000 opioid-related deaths, the greatest number ever reported in a 12-month period. ${ }^{21}$ Many authorities believe that in the United States opioid medications have been overprescribed, contributing to the opioid misuse epidemic. There is little evidence that chronic pelvic pain is optimally managed by chronic treatment with an opioid. ${ }^{22,23}$ Prescribing opioids to vulnerable individuals to treat chronic pelvic pain may result in opioid dependency and adversely affect the patient's health. It is best to pledge not to prescribe an opioid medication for a woman with chronic pelvic pain caused by endometriosis. In situations when pelvic pain is difficult to control with hormonal therapy and nonopioid pain medications, referral to a specialty pain practice may be warranted.

\section{Post-conservative surgery hormone treatment reduces pelvic pain recurrence}

In a meta-analysis of 14 studies that reported on endometriosis recurrence rates following conservative surgery, recurrence (defined as recurrent pelvic pain or an imaging study showing recurrent endometriosis) was significantly reduced with the use of hormone treatment compared with expectant management or placebo treatment. ${ }^{24}$ The postoperative relative risk of endometriosis recurrence was reduced by $83 \%$ with progestin treatment, $64 \%$ with estrogen-progestin contraceptive treatment, and $38 \%$ with GnRH analogue treatment. Overall, the number of patients that needed to be treated to prevent one endometriosis recur- rence was 10, assuming a recurrence rate of $25 \%$ in the placebo treatment or expectant management groups.

For women with pelvic pain caused by endometriosis who develop a recurrence of pelvic pain while on postoperative hormone treatment, it is important for the prescribing clinician to be flexible and consider changing the hormone regimen. For example, if a postoperative patient is treated with a continuous estrogen-progestin contraceptive and develops recurrent pain, I will stop the contraceptive and initiate treatment with either NEA or elagolix.

\section{Capitalize on opportunities to improve the medical care of women with endometriosis}

Early diagnosis of endometriosis can be facilitated by recognizing that the condition is a common cause of moderate to severe dysmenorrhea. In 5 studies involving 1,187 women, the mean length of time from onset of pelvic pain symptoms to diagnosis of endometriosis was 8.6 years. ${ }^{25}$ If a woman with pelvic pain caused by endometriosis has not had sufficient pain relief with one brand of continuous estrogen-progestin contraceptive, it is best not to prescribe an alternative brand but rather to switch to a progestin-only treatment or a GnRH antagonist. If plan A is not working, move expeditiously to plan $\mathrm{B}$.

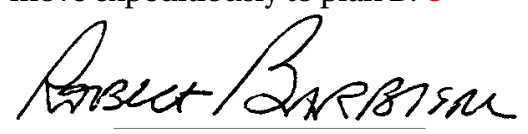

RBARBIERI@MDEDGE.COM

Dr. Barbieri reports no financial relationships relevant to this article.
References

1. Aygestin [package insert]. Barr Laboratories: Pomona, NY; 2007.

2. Kaser DJ, Missmer SA, Berry KF, et al. Use of norethindrone acetate alone for postoperative suppression of endometriosis symptoms. J Pediatr Adolesc Gynecol. 2012;25:105-108.
3. Muneyyirci-Delale O, Karacan M. Effect of norethindrone acetate in the treatment of symptomatic endometriosis. Int J Fertil Womens Med. 1998;43: 
24-27.

4. Muneyyirci-Delale O, Anopa J, Charles C, et al. Medical management of recurrent endometrioma with long-term norethindrone acetate. Int J Women Health. 2012;4:149-154.

5. Morotti M, Venturini PL, Biscaldi E, et al. Efficacy and acceptability of long-term norethindrone acetate for the treatment of rectovaginal endometriosis. Eur J Obstet Gynecol Repro Biol. 2017;213:4-10.

6. Brady PC, Missmer SA, Laufer MR. Hepatic adenomas in adolescents and young women with endometriosis treated with norethindrone acetate. J Pediatr Adolesc Gynecol. 2017;30:422-424.

7. Choudhary NS, Bodh V, Chaudhari S, et al. Norethisterone related drug induced liver injury: a series of 3 cases. J Clin Exp Hepatol. 2017;7:266268.

8. Perez-Mera RA, Shields CE. Jaundice associated with norethindrone acetate therapy. $N$ Engl J Med. 1962;267:1137-1138.

9. Camila [package insert]. Mayne Pharma Inc: Greenville, NC; 2018.

10. Barbieri RL. Elagolix: a new treatment for pelvic pain caused by endometriosis. OBG Manag. 2018;30:10,12-14, 20.

11. Taylor HS, Giudice LC, Lessey BA, et al. Treatment of endometriosis-associated pain with elagolix, an oral GnRH antagonist. $N$ Engl $J$ Med. 2017;377:28-40.

12. Surrey E, Taylor HS, Giudice L, et al. Long-term outcomes of elagolix in women with endometriosis: results from two extension studies. Obstet Gynecol. 2018;132:147-160.

13. Orilissa [package insert]. AbbVie Inc; North Chicago, IL; 2018.

14. Barbieri RL. Hormonal treatment of endometriosis: the estrogen threshold hypothesis. Am J Obstet Gynecol. 1992;166:740-745.

15. Hornstein MD, Surrey ES, Weisberg GW, et al. Leuprolide acetate depot and hormonal add-back in endometriosis: a 12-month study. Lupron Add-Back Study Group. Obstet Gynecol. 1998;91:16-24.

16. Gallagher JS, Missmer SA, Hornstein MD, et al. Long-term effects of gonadotropin-releasing hormone agonists and add-back in adolescent endometriosis. J Pediatr Adolesc Gynecol. 2018;31:376381.

17. Miller A, Shor R, Waites T, et al. Prior authorization reform for better patient care. J Am Coll Cardiol. 2018;71:1937-1939.

18. Depot-leuprolide acetate. Good Rx website. https://www.goodrx.com/. Accessed January 22,
2021.

19. Goserelin. Good $\mathrm{Rx}$ website. https://www .goodrx.com/. Accessed January 22, 2021

20. Taylor HS, Giudice LC, Lessey BAet al. Treatment of endometriosis-associated pain with elagolix, an oral GnRH antagonist. $N$ Engl J Med. 2017;377:28-40.

21. Centers for Disease Control and Prevention Overdose deaths accelerating during COVID19. https://www.cdc.gov/media/releases/2020 /p1218-overdose-deaths-covid-19.html. Reviewed December 18, 2020. Accessed March 24, 2021.

22. Till SR, As-Sanie S. 3 cases of chronic pelvic pain with nonsurgical, nonopioid therapies. $O B G$ Manag. 2018;30:41-48.

23. Steele A. Opioid use and depression in chronic pelvic pain. Obstet Gynecol Clin North Am. 2014;41:491-501.

24. Zakhari A, Delpero E, McKeown S, et al. Endometriosis recurrence following post-operative hormonal suppression: a systematic review and meta-analysis. Hum Reprod Update. 2021;27:96107.

25. Barbieri RL. Why are there delays in the diagnosis of endometriosis? OBG Manag. 2017;29:8, 10-11, 16. 